\title{
Electroluminescence Generation in PbS Quantum Dot Light-Emitting Field-Effect Transistors with Solid-State Gating
}

Artem G. Shulga, ${ }^{\dagger}$ Simon Kahmann, ${ }^{\dagger}$ Dmitry N. Dirin, ${ }^{\ddagger} \S^{\circ}$ Arko Graf, ${ }^{\|}$Jana Zaumseil, ${ }^{\| \odot}$ Maksym V. Kovalenko, ${ }^{\ddagger}, \S$ and Maria A. Loi*,†

${ }^{\dagger}$ Zernike Institute for Advanced Materials, University of Groningen, NL-9747AG Groningen, The Netherlands

${ }^{\ddagger}$ Department of Chemistry and Applied Biosciences, ETH Zürich, CH-8093 Zürich, Switzerland

${ }^{\S}$ Empa-Swiss Federal Laboratories for Materials Science and Technology, CH-8600 Dübendorf, Switzerland

"Institute for Physical Chemistry, Universität Heidelberg, DE-69120 Heidelberg, Germany

Supporting Information

ABSTRACT: The application of light-emitting field-effect transistors (LEFET) is an elegant way of combining electrical switching and light emission in a single device architecture instead of two. This allows for a higher degree of miniaturization and integration in future optoelectronic applications. Here, we report on a LEFET based on lead sulfide quantum dots processed from solution. Our device shows state-of-the-art electronic behavior and emits nearinfrared photons with a quantum yield exceeding $1 \%$ when cooled. We furthermore show how LEFETs can be used to
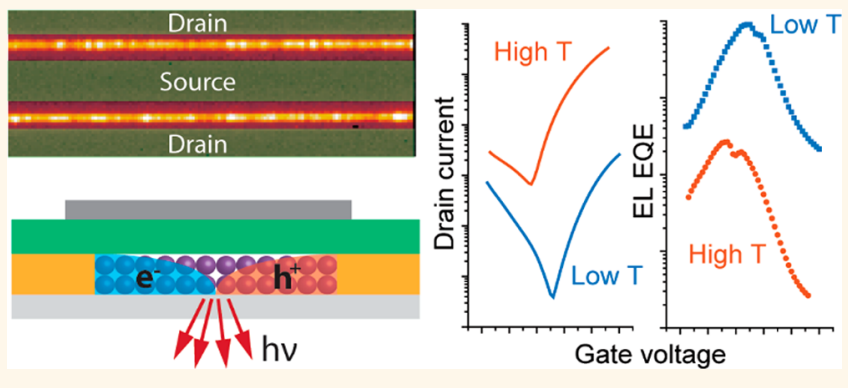
simultaneously characterize the optical and electrical material properties on the same device and use this benefit to investigate the charge transport through the quantum dot film.

KEYWORDS: quantum dots, field-effect transistors, light emission, low temperature, traps

olloidal quantum dots (CQDs), deposited as a thin film, are a modern semiconducting material with attractive optical and electronic properties and have been used in a variety of devices including solar cells, lightemitting diodes (LEDs), photodiodes, field-effect transistors (FETs), and microelectronic circuits. ${ }^{1-6}$ Among the large variety of semiconducting CQDs that have been synthesized over the last years, especially lead chalcogenide, such as $\mathrm{PbS}$ and $\mathrm{PbSe}$, CQDs received large attention because of their easy synthesis, high material quality, and consequent large success as an active layer of different optoelectronic devices. ${ }^{7-9}$ In particular, Pb-based compounds have been dominating the research activities in CQD solar cells, given their large band gap tunability and good transport properties. ${ }^{10-15}$ Solar cell power conversion efficiencies above $11 \%$ were reported, showing the success of a large number of research activities devoted to the improvement of the CQDs' surface passivation $^{16}$ as well as the device structure. ${ }^{1}$ Besides the large interest in solar cells and photodetectors based on CQDs, their broadly tunable optical properties and the good transport properties allow for the fabrication of different types of optoelectronic devices, where light emission in the NIR is required. ${ }^{17}$ NIR light sources, especially when they can be made of nanometer size, can have applications in very different fields, e.g., the biomedical one or visible light communications (VLC). ${ }^{18,19}$

A less explored optoelectronic device is the so-called lightemitting field-effect transistor (LEFET), which simultaneously allows for current switching and electroluminescence generation, potentially capable of taking the roles of both LEDs and FETs in microelectronic circuits. ${ }^{20,21}$ Typically, LEFETs are based on ambipolar FETs, where the source and the drain electrodes are able to inject both electrons and holes, into the transport energy levels of the semiconducting material. There are numerous reports of LEFETs made from organic materials or carbon nanotubes; ${ }^{22-26}$ however, only electrolyte-gated LEFETs made from CQDs (QDLEFET) and an example of CQD-based hybrid LED/LEFETs have been reported recently (for comparison of the performance metrics of CQDcontaining LEFETs see Supplementary Table S1). ${ }^{27-29}$

Received: October 17, 2018

Accepted: December 12, 2018

Published: December 12, 2018 

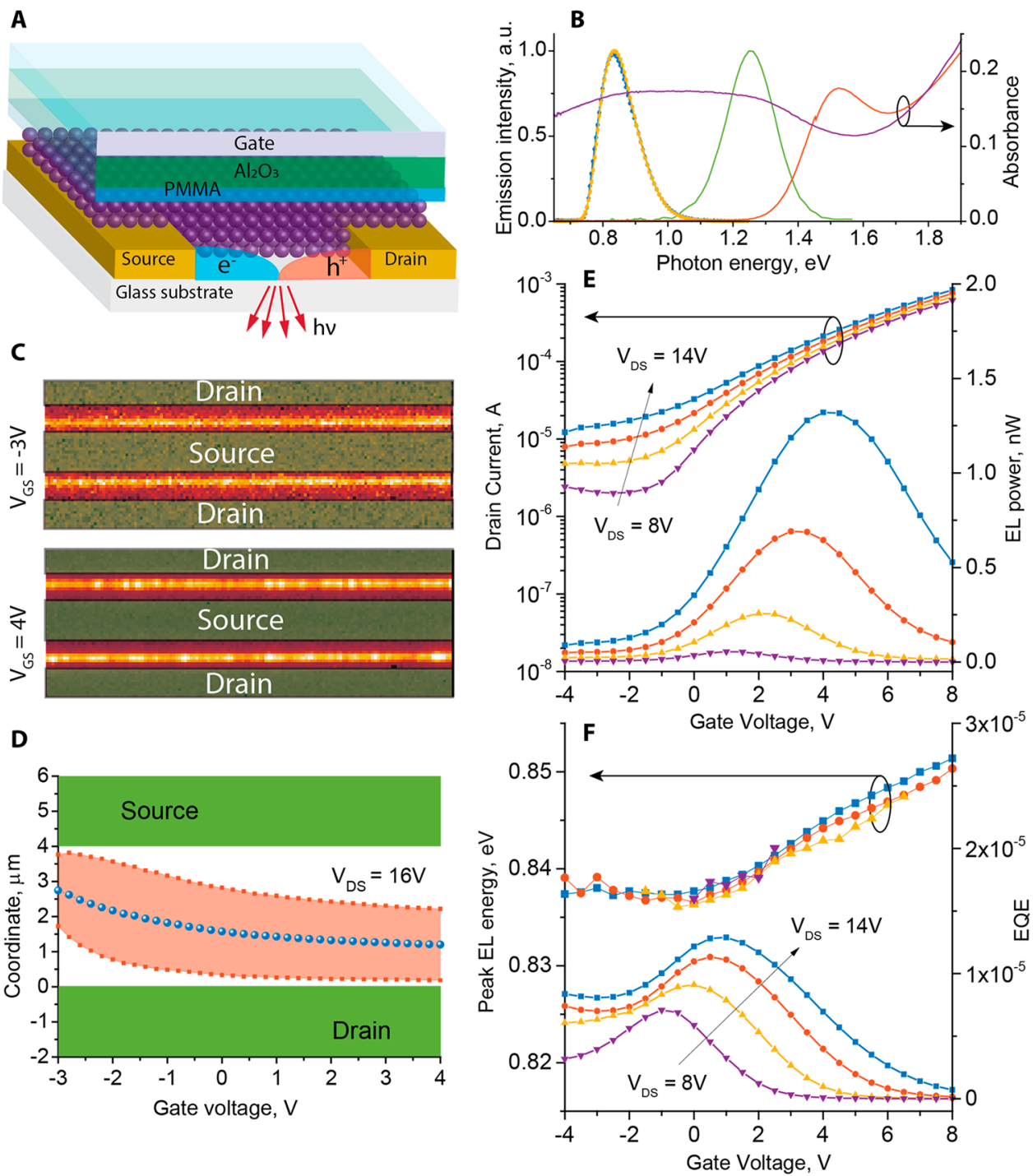

Figure 1. QDLEFET device schematics (A) and electroluminescence generation properties. (B) Absorbance and photoluminescence spectra (red and green curves) of an OA-capped PbS CQDs solution, along with the absorbance, photoluminescence, and electroluminescence spectra (violet, blue, and yellow curves, respectively) of a TBAI-treated PbS CQDs thin film. (C) Images of the QDLEFET channel for the gate voltage of $-3 \mathrm{~V}$ (top) and $4 \mathrm{~V}$ (bottom). (D) Position of the recombination area inside the channel as a function of the gate voltage with the width of the Lorentzian peak (red area). (E) Drain current of the QDLEFET and corresponding electroluminescence power versus the gate voltage. (F) Peak energy of the Gaussian-fitted electroluminescence peak and EQE versus the gate voltage.

Despite showing great performance in various devices, CQD solids require additional studies addressing challenges associated with the nature of the material. Due to their large surface to volume ratio, charge transport in $\mathrm{Pb}$-based CQD FETs is influenced by the CQDs synthesis strategies, device fabrication conditions, the stoichiometry of the surface, and the nature of the ligands. ${ }^{14,30-33}$ For example, electron transport through a CQDs film can be affected by electron trapping from oxygen or water adsorbates, deposited during or after film casting. ${ }^{30}$ The imbalanced stoichiometry of the surface of individual CQDs can strongly affect the carrier mobility. ${ }^{31,34}$ Additionally, the nature of the ligands in the CQDs film can shift their energy levels, affect charge carrier mobility, and introduce additional charge traps, deteriorating the transport. ${ }^{4,35}$

Important information on the trap states and transport mechanism, in general, can be obtained with low-temperature studies. $^{36-38}$ CQD films typically exhibit a phonon-assisted hopping transport, which is expected to become band-like in highly coupled CQD solids. ${ }^{37,39-41}$ Furthermore, phononassisted dissociation of excitons was reported to dominate the temperature-dependent photoluminescence of coupled $\mathrm{PbS}$ CQD films. ${ }^{42}$ Trap states have been also reported to influence the photoluminescence emission of these materials in particular at low temperature due to their shallow nature. ${ }^{43-46}$ Trap-state fingerprints have furthermore been identified in the mid-infrared spectral region in a recent photoinduced absorption study. ${ }^{33}$ The fabrication of CQD-based LEFETs thus not only serves as a demonstration of a very interesting optoelectronic device but also provides a powerful platform to study the transport and material properties of the active layer.

Here we show the first solid-gated purely quantum dotbased LEFET (QDLEFET), made from widely used tetrabutylammonium iodide (TBAI)-treated $\mathrm{PbS}$ CQDs. Infrared electroluminescence with a quantum efficiency above $1 \%$ is obtained for temperatures below $100 \mathrm{~K}$. This 
external quantum efficiency is $c a .4$ orders of magnitude higher than the one at room temperature. A combination of lowtemperature charge transport and electroluminescence generation measurements allows us to determine the presence of hole trap states on the CQDs. Furthermore, we show that measurements of the QDLEFET conductivity are well fitted by a 2D Mott variable range hopping transport for electrons and show a more complex behavior of the hole conductivity, influenced by thermal activation of the hole-trapping states.

\section{RESULTS AND DISCUSSION}

The schematic structure of a QDLEFET is shown in Figure 1A. CQD films were deposited on top of borosilicate glass substrates with prepatterned gold electrodes, to fabricate FETs in bottom contact/top gate configuration. The CQD films were deposited by spin-casting using a layer-by-layer technique with subsequent ligand exchange from the native oleic acid (OA) to TBAI, which is one of the most widely used ligands for fabrication of $\mathrm{PbS}$ optoelectronic devices. ${ }^{1,5,14} \mathrm{~A}$ thin layer of poly(methyl methacrylate) (PMMA) was subsequently spincoated on top of the CQD film, before growing by atomic layer deposition (ALD) of an aluminum oxide layer. Both layers together form the gate dielectric. The use of PMMA decreases the achievable gate capacitance, but prevents interaction between the active layer and the chemically active aluminum precursor during the ALD process. To complete the device, a gold top gate electrode was evaporated through a shadow mask. After fabrication, the devices were annealed to improve the charge transport of the CQD films. The device design allowed for measuring both photoluminescence and electroluminescence from the same CQD film.

An overview of the spectral characteristics of the employed CQDs is given in Figure 1B. The absorption peak for OAcapped CQDs in hexane is found at $1.53 \mathrm{eV}(809 \mathrm{~nm})$, corresponding to a diameter of the QDs of approximately 2.75 $\mathrm{nm} .{ }^{47}$ The photoluminescence is shifted down to $1.26 \mathrm{eV}$ (985 $\mathrm{nm}$ ), thus showing the large Stokes shift typical for such small CQDs of this material. The origin of this Stokes shift still remains debated in the literature and is often attributed to the aggregations of the CQDs. ${ }^{48}$ Cast as a TBAI-treated film after annealing, the CQDs exhibit their maximum PL at an even lower energy of $0.84 \mathrm{eV}(1480 \mathrm{~nm})$, as a consequence of the decreased confinement of the carrier wave function. ${ }^{49}$ The EL, measured from the QDLEFET, almost overlaps with the PL spectrum. The absorbance spectrum demonstrates the first excitonic peak with a broad absorption shoulder in the infrared region, presumably caused by trap states, distributed in the band gap.

In the following, we shall discuss the device characteristics at room temperature (RT), after which we shall consider the behavior upon temperature variation to elucidate the working mechanisms of the fabricated QDLEFETs.

Figure 1C shows two NIR optical micrographs of the electroluminescence between the source and drain electrode of our devices for the cases of a gate voltage $\left(V_{\mathrm{GS}}\right)$ of $-3 \mathrm{~V}$ (top) and $+4 \mathrm{~V}$ (bottom). In the former case, the maximum emission intensity lies closer to the source, while in the latter case it lies closer to the drain electrode. Changing the gate voltage thus shifts the zone of electron-hole recombination between the electrodes through the center of the channel. This demonstrates that in QDLEFETs we can exploit the main advantages of LEFETs compared to LEDs, i.e., the spatial control of the recombination zone and ability to move it away from the electrodes to increase the EL efficiency. ${ }^{50}$ To map this behavior in more detail, we plotted the position of the maximum EL (blue circles) and its full width at half-maximum (red squares) as a function of the gate voltage in Figure 1D $\left(V_{\mathrm{DS}}=16 \mathrm{~V}\right)$. The QDLEFET drain current is shown in Figure $1 \mathrm{E}$ as a function of the gate voltage (transfer curve). When biased in saturation mode (large $V_{\mathrm{DS}}$ ), the QDLEFET shows asymmetric ambipolar characteristics, with the conductivity of the electron channel exceeding the hole conductivity. The emitted electroluminescence was detected with a calibrated photodiode and is also included in Figure 1E. Due to the large difference in the conductivity for electrons and holes, the maximum of the detected EL power is observed for positive gate voltages of approximately 1 to $5 \mathrm{~V}$ (for $V_{\mathrm{DS}}$ from 8 to 14 $\mathrm{V}$ ) and increases with the $V_{\mathrm{DS}}$ due to a higher (drain) current injection. These bias values correspond to the case where the recombination zone is already close to the drain electrode. At larger gate voltage, the photocurrent decreases despite an increasing drain current. This effect is due to both the increase of electrode-mediated nonradiative exciton recombination and the extraction of electrons at the drain electrode. However, as expected, the maximum value of the EL external quantum efficiency (EQE), i.e., the number of emitted photons per transported electron, is observed for the case where the recombination zone lies in the center of the channel (Figure $1 \mathrm{~F}$ and $\mathrm{D}$ ). The absolute value of the EQE (up to $1.3 \times 10^{-5}$ ) increases with the applied drain voltage, presumably due to the filling of trap states in the channel. ${ }^{24,51}$

The shape of the EL spectrum of the fabricated QDLEFETs varies slightly from sample to sample and in most cases can be fitted with a single Gaussian peak. The fitted peak photon energy reaches a minimum of $837 \mathrm{meV}$ for low or negative gate voltages (Figure $1 \mathrm{~F}$ ), but upon increasing $V_{\mathrm{GS}}$, the $\mathrm{EL}$ maximum shifts by $14 \mathrm{meV}$ to $851 \mathrm{meV}$, while being hardly affected by the $V_{\mathrm{Ds}}$. This trend nicely follows the behavior of the drain current, as shown in Figure 1E, and we therefore ascribe it to state filling due to a higher carrier concentration in the channel.

Besides the high electroluminescence quantum efficiency, for being a viable technology, QDLEFETs have to enable electrical switching like conventional FETs. The switching properties of our devices are characterized by the output and transfer curve reported in Figure 2A and B, respectively. The dashed lines in the figure are the backward scans, which for negative gate voltages are substantially different from the forward scan (continuous line), indicating a large hysteresis, as often reported for QD FETs. ${ }^{4,36,52-54}$ The negligible hysteresis for electron accumulation (right plot) opposite the marked effect in the hole channel (left plot) indicates the presence of holetrapping states within the band gap of the CQDs, while electron-trapping states are shallow and cannot be distinguished from the transport states. It should be noted that this nonsymmetric hysteresis in QD FETs has been reported previously by our group and other researchers. ${ }^{5,27,31,54}$ The commonly accepted explanation of the hysteresis is the presence of charge traps, which can be induced by the fabrication process, can be located at the dielectric/semiconductor interface, or can be within the semiconducting active layer. ${ }^{30,55}$ In our case, the samples are fabricated in inert atmosphere to avoid contact with environmental molecules known to be effective electron traps (vide infra); also the dielectric layer, in this case composed of PMMA and $\mathrm{Al}_{2} \mathrm{O}_{3}$, has a less harmful effect (lower interfacial trap density) than 

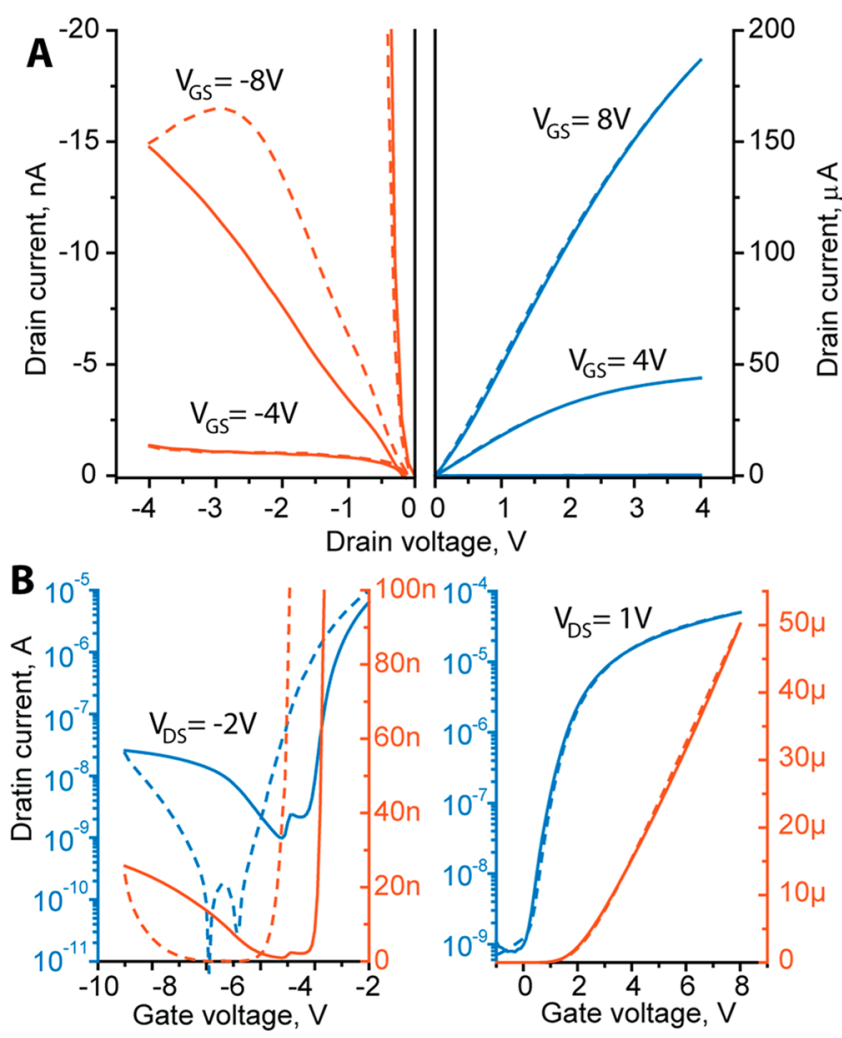

Figure 2. Output (A) and transfer (B) curves of a QDLEFET. The hysteresis is reported for the curves with the forward and the backward scan illustrated by continuous and dashed curves, respectively. The output curves (A) for the hole channel are plotted in the left and for the electron channel in the right plot. The transfer curves (B) are plotted in exponential (left axis, blue curve) and linear (right axis, orange curve) scale for hole channel (left plot) and electron channel (right plot).

oxides such as the most common $\mathrm{SiO}_{2}$. Additionally, the top gate structure serves as an encapsulating layer, ensuring high air stability of the devices. We therefore assume that the traps here predominantly originate from the active material. The nature of such trap states in CQDs was discussed in several reports, and the suggestions include a nonideal surface stoichiometry, uncoordinated dangling bonds, or adsorbates. ${ }^{34,33,56,57}$

The extracted linear electron mobility has a value of 0.06 $\mathrm{cm}^{2} \mathrm{~V}^{-1} \mathrm{~s}^{-1}$ and exhibits a correlation with the gate voltage, in good agreement with the hopping electron transport model through an exponential tail of the density of states. ${ }^{38}$ The large hysteresis of the transfer curves for the hole channel, on the other hand, obscures the hole transport properties and prevents the extraction of meaningful values of the hole mobility. In $\mathrm{n}$-channel operation, the QDLEFET shows an on-off ratio greater than $10^{4}$, and the switching properties of the transistor could be further improved by inserting holeblocking contacts, for example. However, it is important to underline that the transport performance of these transistors are close to state-of-the-art $\mathrm{PbS}$ CQDs devices, reported recently by one of our groups. ${ }^{5}$

To acquire deeper insights into the charge transport and a better understanding of the reasons for the relatively low EL EQE values, we furthermore studied the device properties at low temperatures.
The temperature dependence of the transfer curves of the QDLEFET, measured in saturation mode, and the corresponding EL EQE values are shown in Figure $3 \mathrm{~A}$ and $\mathrm{B}$, respectively.
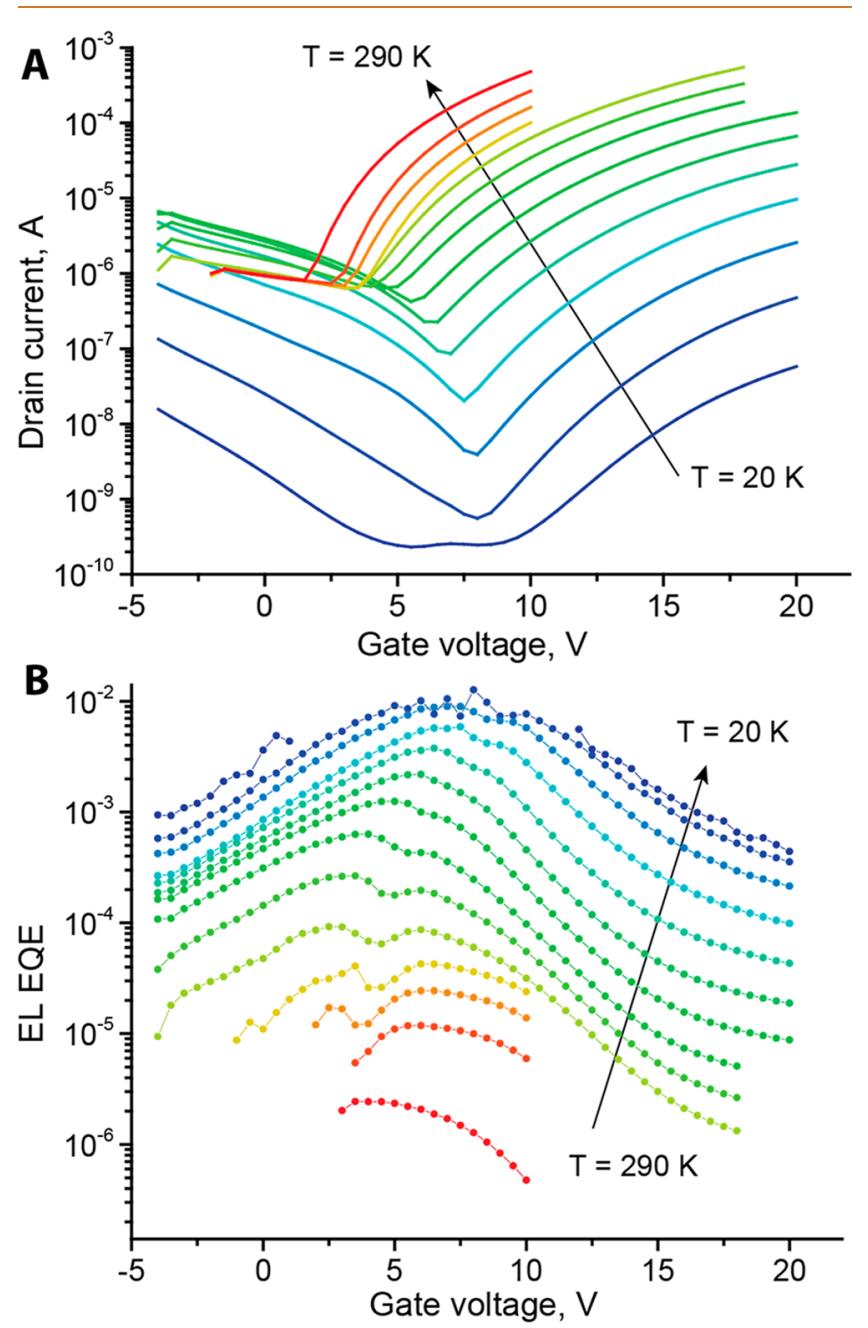

Figure 3. Temperature dependence of the drain current (A) and of the EL EQE (B) versus the gate voltage. Measurements were taken for $V_{\mathrm{DS}}=18 \mathrm{~V}$. The overall drain current decreases at lower temperatures, while the EL EQE increases by 4 orders of magnitude.

The electron current exhibits a monotonic increase with temperature from $20 \mathrm{~K}$ up to $\mathrm{RT}$, which is in good agreement with phonon-assisted hopping charge transport models. The hole current, instead, displays a more complex behavior. It increases analogously to the electron current up to $80 \mathrm{~K}$, reaches a peak at $140 \mathrm{~K}$, and decreases up to $220 \mathrm{~K}$, after which the value remains almost constant up to RT. This behavior can be explained by the competition between thermally activated phonon-assisted hopping transport through the transport states and the charge-trapping process into deeper energy levels, which are also thermally activated. While the first of the processes increases the hole mobility, the second, on contrary, impedes the hole transport. ${ }^{37}$ Dissimilarly to the current, the EL EQE values decrease monotonically with increasing temperature (Figure 3B).

To investigate the charge transport mechanism in QDLEFETs, we performed low-temperature conductivity measurements of the electron and hole channel in the linear 
regime. $^{37}$ The results are depicted in Figure 4A. The conductivity of the electron channel can be fitted very well

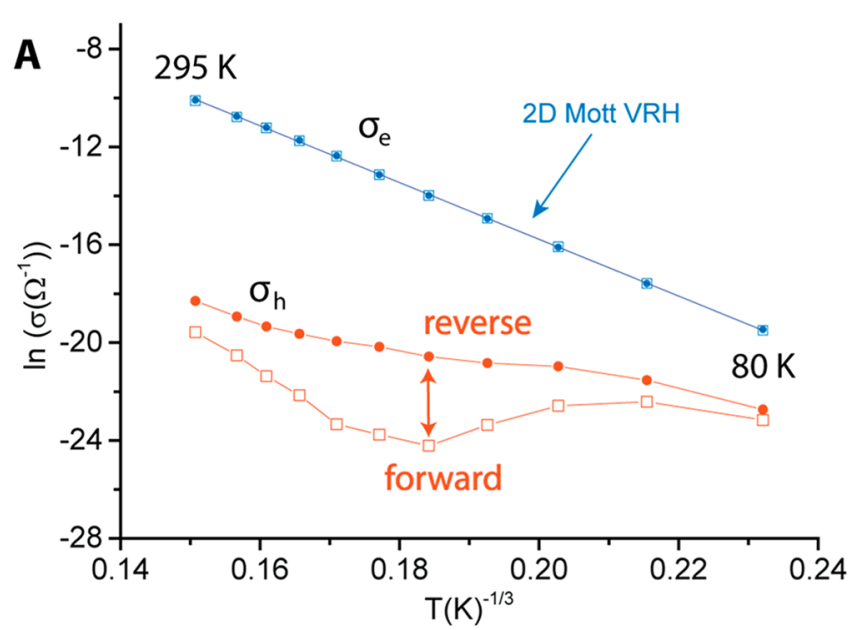

B

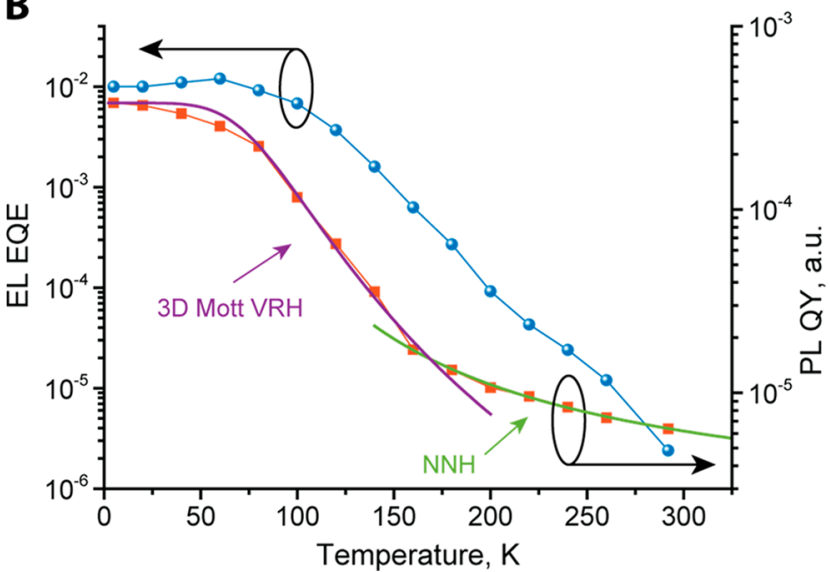

Figure 4. (A) Temperature dependence of electron and hole conductivity in the linear regime (blue and orange lines) plotted versus $T^{-1 / 3}$ according to the $2 \mathrm{D}$ Mott variable range hopping model. The values, extracted from forward and from reverse hysteresis branches, are shown in open squares and in filled circles, respectively. (B) Electroluminescence quantum efficiency and relative quantum yield for photoluminescence versus temperature. The PL curve is fitted using nearest-neighbor hopping ( $\mathrm{NNH})$ at high temperatures and 3D Mott-type variable range hopping (3DMott VRH) at low temperatures.

using the 2D Mott-type variable range hopping model (2D Mott-VRH) ${ }^{58}$ According to the model, the conductivity $\sigma$ is defined by the formula

$$
\sigma=\sigma_{0} \mathrm{e}^{-\left(\frac{T_{\mathrm{Mott}}}{T}\right)^{1 / 3}}
$$

where $\sigma_{0}$ is the conductivity parameter and $T_{\text {Mott }}$ is the characteristic temperature. It is worth mentioning at this point that the general form of Mott-VRH transport includes an exponent $\nu=1 /(1+\mathrm{d})$, with $d$ being the dimensionality of the system (in this case $d=2$ and $\nu=1 / 3$ ).

The straight line fitted to the electron conductivity in Figure 4A has a slope $T_{\text {Mott }}^{1 / 3}=116 \mathrm{~K}^{1 / 3}$, from which the differential temperature-dependent activation energy can be estimated using $\varepsilon_{\mathrm{a}}=\frac{k}{3} T_{\text {Mott }}{ }^{1 / 3} T^{2 / 3}{ }^{59}$ This yields $\varepsilon_{\mathrm{a}}=148 \mathrm{meV}$ at RT, 98 $\mathrm{meV}$ at an intermediate temperature of $160 \mathrm{~K}$, and $25 \mathrm{meV}$ at a low temperature of $20 \mathrm{~K}$. Hopping conductance involves those energy levels located close to the Fermi level in the material, and this range decreases with the temperature, resulting in a decrease of the number of states available for hopping. This is the reason behind temperature dependence of the activation energy, what is not the case in nearest-neighbor hopping (NNH) transport. In contrast to the reported data on ordered superlattices of CQDs, we did not observe switching from nearest-neighbor to variable-range hopping, which could be explained by a larger degree of energetic disorder in our CQD films. $^{38}$

The hole conductivity, measured in the linear regime, is plotted along with the electron conductivity in Figure 4A. The values were taken for both the forward and reverse scanning direction and display a pronounced hysteresis. The hysteresis increases for temperatures higher than $80 \mathrm{~K}$, reaching a maximum between 160 and $200 \mathrm{~K}$, and decreases slightly up to RT. As mentioned above, this effect hinders the determination of the true conductivity and prevents fitting the data using transport theories. We assume that the hole transport can be described by the competition of carrier hopping through transport states and trapping into deeper levels within the fundamental band gap. At low temperatures (below $80 \mathrm{~K}$ ), these trap states are filled, and only a negligible hysteresis is observed. At intermediate temperatures (between 80 and 160 $\mathrm{K})$, thermal detrapping of carriers sets in and the voltage sweep leads to a filling/emptying of the trap states, thus the hysteresis. Furthermore, at high temperatures (exceeding 160 $\mathrm{K})$ the holes acquire enough thermal energy to enter and leave the trap states in the band gap, and the hole conductivity increases with temperature, as is predicted by the hopping model.

Inversely to the conductivity, as shown in Figure 4B, the overall EL EQE increases drastically-by more than 4 orders of magnitude at low temperature-and exceeds $1 \%$ below 100 $\mathrm{K}$. Below $220 \mathrm{~K}$, the emission is strong enough to be detected over the whole gate voltage range. At lower temperature (20 $\mathrm{K})$, the emission from the central part of the channel was not detectable anymore due to the low current. As a side note, we have to state that the device chosen for low-temperature measurements was annealed less $\left(120{ }^{\circ} \mathrm{C}, 30 \mathrm{~min}\right)$ than the one presented in Figure 1. In this way, we could avoid an excessive red-shift of the EL that, in this specific case, was peaked at $0.88 \mathrm{eV}$ at RT. As a consequence, this sample had a reduced maximum RT EL EQE $\left(2.5 \times 10^{-6}\right)$ compared to 1.3 $\times 10^{-5}$ for the samples, which were annealed at higher temperature and longer $\left(130^{\circ} \mathrm{C}, 1 \mathrm{~h}\right)$.

Figure $4 \mathrm{~B}$ shows the temperature dependence of the maximum EQE of the EL and the relative PL quantum yield (the latter given by the ratio of collected photons per incident photons). The EL EQE can be defined as

$$
\eta_{\mathrm{EL}}\left(T, V_{\mathrm{G}}\right)=\gamma\left(T, V_{\mathrm{G}}\right) \eta_{\mathrm{PL}}(T) \chi \sigma_{\text {out }}
$$

where $\gamma$ is the ratio of the exciton formation events with respect to the number of charge carriers injected into the channel, $\eta_{\mathrm{PL}}$ is the photoluminescence quantum efficiency, $\chi$ is the spin multiplicity of the radiatively recombining excitons, and $\sigma_{\text {out }}$ is the light out-coupling efficiency from the device into the open space.

The temperature-dependent photoluminescence quantum efficiency $\eta_{\mathrm{PL}}$ has previously been used to gain insight into the charge carrier transport in CQD thin films. Gao et al. considered the probability of radiative recombination to be in competition with nonradiative recombination and phonon- 

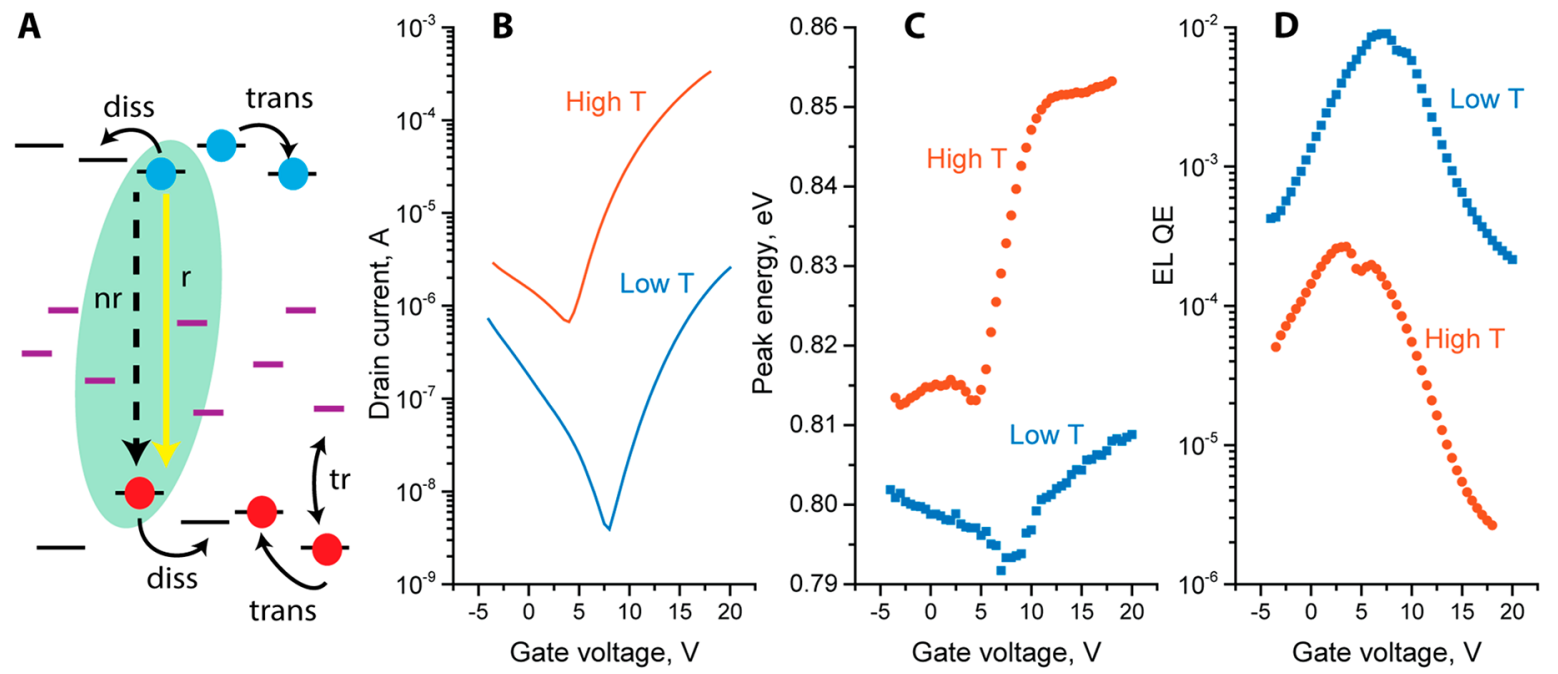

Figure 5. (A) Schematic of the relevant energy levels in the CQD film indicating charge transport ("trans"), transition to hole trap states ("tr"), exciton (the green ellipse), dissociation ("diss"), and exciton radiative/nonradiative recombination ("r" and "nr"). (B) Transfer characteristics of the device at high $(180 \mathrm{~K})$ and low temperature $(80 \mathrm{~K})$. (C) Electroluminescence peak energy versus gate voltage for the two temperatures as in panel B and (D) EL EQE of QDLEFET for the temperatures as in panel $B$.

assisted exciton dissociation; following their discussion, the PL EQE can be defined as ${ }^{42}$

$$
\eta_{\mathrm{PL}}(T)=\eta_{\mathrm{PL}}^{\prime} / 1+\frac{k_{\text {diss }}(T)}{k_{\mathrm{r}}}
$$

where $\eta_{\text {PL }}^{\prime}$ is the efficiency of exciton recombination at $0 \mathrm{~K}$, when the exciton dissociation process is frozen, $k_{\mathrm{r}}$ is the radiative decay rate, and $k_{\text {diss }}(T)$ is the exciton dissociation rate constant of the general form $k_{\text {diss }}(T)=A \mathrm{e}^{-\left(\frac{T_{0}}{T}\right)^{\nu}}$ (consider Figure $5 \mathrm{~A}$ for a scheme). Under the assumption that both $k_{\mathrm{r}}$ and $k_{\mathrm{nr}}$ are temperature independent, they found that no single value of $\nu$ can adequately describe the data across the entire temperature region and fitted the data with $\nu=1$ at high temperature $(\mathrm{NNH})$, with the transition to $\nu=0.5$ (EfrosShklovskii variable range hopping, ES VRH) at low temperature. It should be noted that fitting temperature-dependent PL data, as shown in Figure 4B, does not allow for firmly distinguishing between various values of $\nu$ and, accordingly, to make a conclusion about the hopping mechanism. Similarly to Gao's work, we were unable to find a single value $\nu$ and we fitted the PL EQE data using the $\mathrm{NNH}$ model with the transition to $\mathrm{VRH}$ at low temperatures. $\mathrm{NNH}$ yields an activation energy $\varepsilon_{\mathrm{a}}=31 \mathrm{meV}$ with the crossover temperature $T_{c}=160 \mathrm{~K}$. For the low-temperature part, we based our choice of $\nu$ on the conductivity studies and used the Mott-VRH model (but in three dimensions, since excitons in the PL studies are free to move in the entire film), i.e., with $\nu=0.25$. We find a fitting parameter $T_{0}^{1 / 4}=61.8 \mathrm{~K}^{1 / 4}$. Similarly to the conductivity, the differential activation energy was estimated using the expression $\varepsilon_{\mathrm{a}}=(k / 4) T_{0}^{1 / 4} \mathrm{~T}^{3 / 4}$, giving $60 \mathrm{meV}$ at the crossover temperature $(160 \mathrm{~K})$ and $9 \mathrm{meV}$ at low temperature $(20 \mathrm{~K})$. These values are considerably lower than the activation energy for the electron transport in a $2 \mathrm{D}$ layer, extracted from the conductivity measurements ( $148 \mathrm{meV}$ for RT, $98 \mathrm{meV}$ for $160 \mathrm{~K}$, and $25 \mathrm{meV}$ for $20 \mathrm{~K}$ ).

Based on the discussion above, the temperature dependence of the EL EQE is proportional to

$$
\eta_{\mathrm{EL}}\left(T, V_{\mathrm{G}}\right) \approx \gamma\left(T, V_{\mathrm{G}}\right) /\left(k_{\mathrm{r}}+k_{\mathrm{diss}}(T)\right)
$$

For temperatures higher than $100 \mathrm{~K}$ the highest measured EL EQE drastically decreases from $1 \%$ down to $0.001 \%$, showing a sharp temperature dependence as well as the PL EQE. The EL EQE temperature dependence is defined by the exciton dissociation, which are neutral species and are not affected by the electric field; therefore, we expect it to be governed as the PL by $\mathrm{NNH}$ and $3 \mathrm{D} \mathrm{VRH}$ rather than $2 \mathrm{D}$ Mott-VRH, as found for the conductivity. However, as mentioned for the PL EQE and shown in the Supporting Information (Figure S1), fitting our set of data does not allow making a firm conclusion about the type of hopping based on the exponent. Additionally, these hopping theories do not take the presence of hole trapping energy levels into account, the presence of which was evident from the conductivity studies.

Figure 5 illustrates the mechanism of carrier conduction, exciton dissociation, and electroluminescence generation in QDLEFETs. In the schematics in Figure 5A, the transport energy levels of the CQDs film are represented by black lines, showing the energetic disorder determined by the assembly of the individual dots, and hole-trapping states are shown by violet lines. Excitons, which are indicated here with the green ellipse, are formed after charge capture and can undergo radiative/nonradiative recombination or dissociation (indicated with arrows and letters).

It is important to note that in the spectral range investigated we do not observe optical transitions from/to trap states; the main significant influence of the charge trapping is the decrease of the hole mobility and the appearance of the hysteresis.

The influence of the hole trapping on the transfer curves of the QDLEFET at two characteristic temperatures is illustrated in Figure 5B. For low temperature $(80 \mathrm{~K})$, the curve has a symmetrical "V"-shape, representing similar charge transport through transport levels for electrons and holes. As stated above, these traps are thus inactive (filled) at low temperature. However, for higher temperatures $(180 \mathrm{~K})$ the detrapping process is activated and the shape of the transfer curve becomes asymmetrical. 
Figure 5C shows the dependence of the energy of the electroluminescence peak versus the applied gate voltage. The gate voltage effectively shifts the Fermi level in the QDLEFET, moving it deeper into charge transport levels, therefore including more states for electron or hole transport. This leads to a band filling and a blue shift of the emission for larger applied voltages at low temperature (15 and $8 \mathrm{meV}$ for electron and hole accumulation, respectively). However, for high temperatures, the emission blue-shifts much more noticeably for the electron channel than for the hole channel. In the former case, the shift amounts to $38 \mathrm{meV}$, but does not exceed $2 \mathrm{meV}$ in the latter. Given the activation of the trap states at elevated temperatures, we expect the Fermi level to be pinned to the hole trap level at a negative bias, which effectively prevents shifting it deeper into the hole transport states. Therefore, the emission does not further blue-shift, but the energy is limited by the energetic position of the trap states. We furthermore note that the peak energy of the minimum emission energy is reduced by $19 \mathrm{meV}$ at low temperature, which is predominantly the result of the band gap decrease of $\mathrm{PbS}$ CQDs at low temperature. ${ }^{44,61}$

As discussed above, the phonon-assisted exciton dissociation leads to the inverse dependence of the $\mathrm{EL} \mathrm{EQE}$ on the temperature: it decreases from $0.9 \%$ at $80 \mathrm{~K}$ to $0.026 \%$ at 180 $\mathrm{K}$. However, the shape of the EL EQE curve as a function of the gate voltage remains similar, with a small shift of the peak toward larger gate bias for the low-temperature case. The EL EQE decreases symmetrically for large positive/negative gate voltage both at high and low temperature, indicating that when the recombination zone is close to either of the electrodes, the influence of the contacts is limiting the EL EQE rather than the charge-trapping process.

\section{CONCLUSIONS}

In conclusion, we presented and characterized the first fully solid QDLEFET and showed how this device not only has interesting application prospects modulating simultaneously optical and electrical signals but can also be used to study the physical properties of CQD films. The QDLEFET shows good switching properties as an n-type FET, with hysteresis-free electron transport giving a mobility of $0.06 \mathrm{~cm}^{2} \mathrm{~V}^{-1} \mathrm{~s}^{-1}$. The hole transport shows a lower mobility and a pronounced hysteresis, pointing to the presence of hole traps in the CQDs. Measurements of the QDLEFET conductivity are well fitted by a 2D Mott-VRH transport for electrons and show a more complex behavior of the hole conductivity, influenced by thermal activation of the hole-trapping energy states. By changing the gate bias, the recombination zone can be shifted within the channel of the transistor from source or drain electrode to the middle of the channel, where the EL EQE is significantly increased. At RT, a maximal EL EQE of $1.3 \times$ $10^{-5}$ is obtained. The emission peak energy depends on the gate voltage and can be shifted on the order of $10 \mathrm{meV}$, an effect we ascribe to state filling of the transport levels at higher carrier densities. The EL EQE increases drastically at low temperatures, reaching $1 \%$ below $100 \mathrm{~K}$. This behavior is explained by phonon-assisted dissociation of excitons.

\section{MATERIALS AND METHODS}

PbS CQDs Synthesis. Lead(II) acetate trihydrate ( $\geq 99.99 \%$, Aldrich), bis(trimethylsilyl)sulfide (Aldrich), 1-octadecene (ODE, $90 \%$, Aldrich), oleic acid (90\%, Aldrich), ethanol (Fluka), hexane (Aldrich), and tetrachloroethylene (99\%, Aldrich) were used as received. $\mathrm{PbS}$ CQDs capped with oleate ligands were synthesized as described elsewhere with slight modifications. ${ }^{5}$ A $1.5 \mathrm{~g}$ amount of lead(II) acetate trihydrate was dissolved in a mixture of $47.2 \mathrm{~mL}$ of ODE and $2.8 \mathrm{~mL}$ of oleic acid. This solution was dried for $1 \mathrm{~h}$ under vacuum at $120{ }^{\circ} \mathrm{C}$ in a three-neck flask using a Schlenk line. Further reaction was carried out under an argon atmosphere. The lead precursor solution was cooled to $85{ }^{\circ} \mathrm{C}$, the heating mantle was removed, and a solution of $0.420 \mathrm{~mL}$ of bis(trimethylsilyl)sulfide in 10 $\mathrm{mL}$ of dried ODE was quickly injected. Two minutes later the reaction was quenched using a cold water bath. CQDs were purified three times by washing with a hexane/ethanol mixture. Finally, $\mathrm{PbS}$ CQDs were redispersed in anhydrous hexane, filtered through a 450 $\mu \mathrm{m}$ PTFE filter, and stored under an inert atmosphere. Solution concentrations were determined by the measurement of the absorption of diluted solutions at $400 \mathrm{~nm}$.

Device Fabrication. A borosilicate glass plate $(0.7 \mathrm{~mm}$ thick $)$ was chosen as the substrate. After cleaning, the source and drain electrodes $(\mathrm{Ti} / \mathrm{Au}, 5 / 40 \mathrm{~nm})$ were patterned using standard lift-off lithography technique. After resist removal, the substrate was annealed at $120{ }^{\circ} \mathrm{C}$ in a $\mathrm{N}_{2}$-filled glovebox. The deposition of a CQD film took place in the glovebox, shortly after annealing. The film was completed by spin-coating OA-capped PbS CQDs $(2 \mathrm{mg} / \mathrm{mL}$ for the first layer and $20 \mathrm{mg} / \mathrm{mL}$ for the next three layers in hexane $)^{4}$ and subsequent treatment of the layers using TBAI $(11 \mathrm{mg} / \mathrm{mL}$ in methanol) for $c a$. $35 \mathrm{~s}$. After ligand exchange, the layers were washed twice with pure methanol. After the completion of the CQD film, the substrate was dried on a hot plate for $1 \mathrm{~min}$ at $120^{\circ} \mathrm{C}$. Then, an approximately 10 $\mathrm{nm}$ thick PMMA interlayer was spin-coated $(10 \mathrm{mg} / \mathrm{mL}$ in toluene). The substrate was transferred to the ALD chamber, and 600 cycles of aluminum oxide were grown at $100{ }^{\circ} \mathrm{C}$. Lastly, a $100 \mathrm{~nm}$ thick gold gate electrode was thermally evaporated through a shadow mask.

Device Characterization. Electrical characterization of the QDLEFET was done using a Keithley 4200-SCS semiconductor parameter analyzer. Electroluminescence and photoluminescence spectra were collected using a spectrometer and recorded by an Andor iDus $1.7 \mu \mathrm{m}$ InGaAs camera. The electroluminescence EQE was calculated using a calibrated photodiode, put in contact with the back side of the substrate. Using these data, the camera was calibrated to estimate the EQE of the electroluminescence and photoluminescence at low temperature. For low-temperature measurements, the substrate was placed in a He-cooled cryostat with springloaded contact pins for reliable electrical connection. Channel imaging was done by a cooled $2 \mathrm{D}$ InGaAs camera $(640 \times 512$ pixels NIRvana 640ST, Princeton Instruments), using a 50× objective.

\section{ASSOCIATED CONTENT}

\section{Supporting Information}

The Supporting Information is available free of charge on the ACS Publications website at DOI: 10.1021/acsnano.8b07938.

Fitting EL EQE temperature dependence with different hopping models; comparison of the performance metrics for CQD-containing LEFETs (PDF)

\section{AUTHOR INFORMATION}

\section{Corresponding Author}

*Phone: +31 50363 4119. Fax: +31 50363 8751. E-mail: M.A. Loi@rug.nl.

\section{ORCID}

Simon Kahmann: 0000-0001-7784-5333

Dmitry N. Dirin: 0000-0002-5187-4555

Jana Zaumseil: 0000-0002-2048-217X

Maksym V. Kovalenko: 0000-0002-6396-8938

Maria A. Loi: 0000-0002-7985-7431

Notes

The authors declare no competing financial interest. 


\section{ACKNOWLEDGMENTS}

The Groningen team acknowledges the financial support of the European Research Council (ERC) starting grant (No. 306983) "Hybrid solution processable materials for optoelectronic devices” (ERC-HySPOD).

\section{REFERENCES}

(1) Speirs, M. J.; Dirin, D. N.; Abdu-Aguye, M.; Balazs, D. M.; Kovalenko, M. V.; Loi, M. A. Temperature Dependent Behaviour of Lead Sulfide Quantum Dot Solar Cells and Films. Energy Environ. Sci. 2016, 9, 2916-2924.

(2) Sun, L.; Choi, J. J.; Stachnik, D.; Bartnik, A. C.; Hyun, B.-R.; Malliaras, G. G.; Hanrath, T.; Wise, F. W. Bright Infrared QuantumDot Light-Emitting Diodes through Inter-Dot Spacing Control. Nat. Nanotechnol. 2012, 7, 369-373.

(3) McDonald, S. A.; Konstantatos, G.; Zhang, S.; Cyr, P. W.; Klem, E. J. D.; Levina, L.; Sargent, E. H. Solution-Processed PbS Quantum Dot Infrared Photodetectors and Photovoltaics. Nat. Mater. 2005, 4, 138.

(4) Shulga, A. G.; Piveteau, L.; Bisri, S. Z.; Kovalenko, M. V.; Loi, M. A. Double Gate $\mathrm{PbS}$ Quantum Dot Field-Effect Transistors for Tuneable Electrical Characteristics. Adv. Electron. Mater. 2016, 2, 1500467.

(5) Shulga, A. G.; Derenskyi, V.; Salazar-Rios, J. M.; Dirin, D. N.; Fritsch, M.; Kovalenko, M. V.; Scherf, U.; Loi, M. A. An All-SolutionBased Hybrid CMOS-Like Quantum Dot/Carbon Nanotube Inverter. Adv. Mater. 2017, 29, 1701764.

(6) Kim, D. K.; Lai, Y.; Diroll, B. T.; Murray, C. B.; Kagan, C. R. Flexible and Low-Voltage Integrated Circuits Constructed from HighPerformance Nanocrystal Transistors. Nat. Commun. 2012, 3, 1216.

(7) Maulu, A.; Rodríguez-Cantó, P. J.; Navarro-Arenas, J.; Abargues, R.; Sánchez-Royo, J. F.; García-Calzada, R.; Pastor, J. P. M. StronglyCoupled PbS QD Solids by Doctor Blading for IR Photodetection. RSC Adv. 2016, 6, 80201-80212.

(8) Xu, J.; Voznyy, O.; Liu, M.; Kirmani, A. R.; Walters, G.; Munir, R.; Abdelsamie, M.; Proppe, A. H.; Sarkar, A.; Arquer, F. P. G. de; Wei, M.; Sun, B.; Liu, M.; Ouellette, O.; Quintero-Bermudez, R.; Li, J.; Fan, J.; Quan, L.; Todorovich, P.; Tan, H.; et al. 2D Matrix Engineering for Homogeneous Quantum Dot Coupling in Photovoltaic Solids. Nat. Nanotechnol. 2018, 13, 456-462.

(9) Gong, X.; Yang, Z.; Walters, G.; Comin, R.; Ning, Z.; Beauregard, E.; Adinolfi, V.; Voznyy, O.; Sargent, E. H. Highly Efficient Quantum Dot Near-Infrared Light-Emitting Diodes. Nat. Photonics 2016, 10, 253-257.

(10) Tang, J.; Kemp, K. W.; Hoogland, S.; Jeong, K. S.; Liu, H.; Levina, L.; Furukawa, M.; Wang, X.; Debnath, R.; Cha, D.; Chou, K. W.; Fischer, A.; Amassian, A.; Asbury, J. B.; Sargent, E. H. ColloidalQuantum-Dot Photovoltaics Using Atomic-Ligand Passivation. Nat. Mater. 2011, 10, 765-771.

(11) Chuang, C.-H. M.; Brown, P. R.; Bulović, V.; Bawendi, M. G. Improved Performance and Stability in Quantum Dot Solar Cells through Band Alignment Engineering. Nat. Mater. 2014, 13, 796801.

(12) Ning, Z.; Voznyy, O.; Pan, J.; Hoogland, S.; Adinolfi, V.; Xu, J.; Li, M.; Kirmani, A. R.; Sun, J.-P.; Minor, J.; Kemp, K. W.; Dong, H.; Rollny, L.; Labelle, A.; Carey, G.; Sutherland, B.; Hill, I.; Amassian, A.; Liu, H.; Tang, J.; et al. Air-Stable n-Type Colloidal Quantum Dot Solids. Nat. Mater. 2014, 13, 822-828.

(13) Piliego, C.; Protesescu, L.; Bisri, S. Z.; Kovalenko, M. V.; Loi, M. A. 5.2\% Efficient PbS Nanocrystal Schottky Solar Cells. Energy Environ. Sci. 2013, 6, 3054-3059.

(14) Wang, Y.; Lu, K.; Han, L.; Liu, Z.; Shi, G.; Fang, H.; Chen, S.; Wu, T.; Yang, F.; Gu, M.; Zhou, S.; Ling, X.; Tang, X.; Zheng, J.; Loi, M. A.; Ma, W. In Situ Passivation for Efficient PbS Quantum Dot Solar Cells by Precursor Engineering. Adv. Mater. 2018, 30, 1704871.

(15) Bi, Y.; Pradhan, S.; Gupta, S.; Akgul, M. Z.; Stavrinadis, A.; Konstantatos, G. Infrared Solution-Processed Quantum Dot Solar
Cells Reaching External Quantum Efficiency of $80 \%$ at $1.35 \mathrm{Mm}$ and Jsc in Excess of 34 MA Cm-2. Adv. Mater. 2018, 30, 1704928.

(16) Cao, Y.; Stavrinadis, A.; Lasanta, T.; So, D.; Konstantatos, G. The Role of Surface Passivation for Efficient and Photostable PbS Quantum Dot Solar Cells. Nat. Energy 2016, 1, 16035.

(17) Supran, G. J.; Song, K. W.; Hwang, G. W.; Correa, R. E.; Scherer, J.; Dauler, E. A.; Shirasaki, Y.; Bawendi, M. G.; Bulović, V. High-Performance Shortwave-Infrared Light-Emitting Devices Using Core-Shell (PbS-CdS) Colloidal Quantum Dots. Adv. Mater. 2015, 27, 1437-1442.

(18) Benayas, A.; Ren, F.; Carrasco, E.; Marzal, V.; del Rosal, B.; Gonfa, B. A.; Juarranz, Á.; Sanz-Rodríguez, F.; Jaque, D.; García-Solé, J.; Ma, D.; Vetrone, F. PbS/CdS/ZnS Quantum Dots: A Multifunctional Platform for In Vivo Near-Infrared Low-Dose Fluorescence Imaging. Adv. Funct. Mater. 2015, 25, 6650-6659.

(19) Rajbhandari, S.; Chun, H.; Faulkner, G.; Cameron, K.; Jalajakumari, A. V. N.; Henderson, R.; Tsonev, D.; Ijaz, M.; Chen, Z.; Haas, H.; Xie, E.; McKendry, J. J. D.; Herrnsdorf, J.; Gu, E.; Dawson, M. D.; O’Brien, D. High-Speed Integrated Visible Light Communication System: Device Constraints and Design Considerations. IEEE J. Sel. Areas Commun. 2015, 33, 1750-1757.

(20) Zhang, C.; Chen, P.; Hu, W. Organic Light-Emitting Transistors: Materials, Device Configurations, and Operations. Small 2016, 12, 1252-1294.

(21) Liu, C.-F.; Liu, X.; Lai, W.-Y.; Huang, W. Organic LightEmitting Field-Effect Transistors: Device Geometries and Fabrication Techniques. Adv. Mater. 2018, 1, 1802466.

(22) Roelofs, W. S. C.; Adriaans, W. H.; Janssen, R. A. J.; Kemerink, M.; de Leeuw, D. M. Light Emission in the Unipolar Regime of Ambipolar Organic Field-Effect Transistors. Adv. Funct. Mater. 2013, 23, 4133-4139.

(23) Cicoira, F.; Santato, C.; Dadvand, A.; Harnagea, C.; Pignolet, A.; Bellutti, P.; Xiang, Z.; Rosei, F.; Meng, H.; Perepichka, D. F. Environmentally Stable Light Emitting Field Effect Transistors Based on 2-(4-Pentylstyryl)Tetracene. J. Mater. Chem. 2007, 18, 158-161.

(24) Zaumseil, J.; McNeill, C. R.; Bird, M.; Smith, D. L.; Paul Ruden, P.; Roberts, M.; McKiernan, M. J.; Friend, R. H.; Sirringhaus, H. Quantum Efficiency of Ambipolar Light-Emitting Polymer FieldEffect Transistors. J. Appl. Phys. 2008, 103, 064517.

(25) Jakubka, F.; Backes, C.; Gannott, F.; Mundloch, U.; Hauke, F.; Hirsch, A.; Zaumseil, J. Mapping Charge Transport by Electroluminescence in Chirality-Selected Carbon Nanotube Networks. ACS Nano 2013, 7, 7428-7435.

(26) Graf, A.; Held, M.; Zakharko, Y.; Tropf, L.; Gather, M. C.; Zaumseil, J. Electrical Pumping and Tuning of Exciton-Polaritons in Carbon Nanotube Microcavities. Nat. Mater. 2017, 16, 911-917.

(27) Schornbaum, J.; Zakharko, Y.; Held, M.; Thiemann, S.; Gannott, F.; Zaumseil, J. Light-Emitting Quantum Dot Transistors: Emission at High Charge Carrier Densities. Nano Lett. 2015, 15, $1822-1828$

(28) He, P.; Jiang, C.; Lan, L.; Sun, S.; Li, Y.; Gao, P.; Zhang, P.; Dai, X.; Wang, J.; Peng, J.; Cao, Y. High-Performance, SolutionProcessed Quantum Dot Light-Emitting Field-Effect Transistors with a Scandium-Incorporated Indium Oxide Semiconductor. ACS Nano 2018, 12, 4624-4629.

(29) Liu, X.; Kuang, W.; Ni, H.; Tao, Z.; Chang, J.; Liu, Q.; Ge, J.; Li, C.; Dai, Q. High Efficiency Light-Emitting Transistor with Vertical Metal-Oxide Heterostructure. Small 2018, 14, 1800265.

(30) Balazs, D. M.; Nugraha, M. I.; Bisri, S. Z.; Sytnyk, M.; Heiss, W.; Loi, M. A. Reducing Charge Trapping in PbS Colloidal Quantum Dot Solids. Appl. Phys. Lett. 2014, 104, 112104.

(31) Balazs, D. M.; Bijlsma, K. I.; Fang, H.-H.; Dirin, D. N.; Döbeli, M.; Kovalenko, M. V.; Loi, M. A. Stoichiometric Control of the Density of States in PbS Colloidal Quantum Dot Solids. Sci. Adv. 2017, 3, eaao1558.

(32) Oh, S. J.; Berry, N. E.; Choi, J.-H.; Gaulding, E. A.; Paik, T.; Hong, S.-H.; Murray, C. B.; Kagan, C. R. Stoichiometric Control of Lead Chalcogenide Nanocrystal Solids to Enhance Their Electronic 
and Optoelectronic Device Performance. ACS Nano 2013, 7, 24132421.

(33) Kahmann, S.; Sytnyk, M.; Schrenker, N.; Matt, G. J.; Spiecker, E.; Heiss, W.; Brabec, C. J.; Loi, M. A. Revealing Trap States in Lead Sulphide Colloidal Quantum Dots by Photoinduced Absorption Spectroscopy. Adv. Electron. Mater. 2018, 4, 1700348.

(34) Kim, D.; Kim, D.-H.; Lee, J.-H.; Grossman, J. C. Impact of Stoichiometry on the Electronic Structure of $\mathrm{PbS}$ Quantum Dots. Phys. Rev. Lett. 2013, 110, 196802.

(35) Harris, R. D.; Bettis Homan, S.; Kodaimati, M.; He, C.; Nepomnyashchii, A. B.; Swenson, N. K.; Lian, S.; Calzada, R.; Weiss, E. A. Electronic Processes within Quantum Dot-Molecule Complexes. Chem. Rev. 2016, 116, 12865-12919.

(36) Han, L.; Balazs, D. M.; Shulga, A. G.; Abdu-Aguye, M.; Ma, W.; Loi, M. A. PbSe Nanorod Field-Effect Transistors: Room- and LowTemperature Performance. Adv. Electron. Mater. 2018, 4, 1700580.

(37) Guyot-Sionnest, P. Electrical Transport in Colloidal Quantum Dot Films. J. Phys. Chem. Lett. 2012, 3, 1169-1175.

(38) Whitham, K.; Yang, J.; Savitzky, B. H.; Kourkoutis, L. F.; Wise, F.; Hanrath, T. Charge Transport and Localization in Atomically Coherent Quantum Dot Solids. Nat. Mater. 2016, 15, nmat4576.

(39) Choi, J.-H.; Fafarman, A. T.; Oh, S. J.; Ko, D.-K.; Kim, D. K.; Diroll, B. T.; Muramoto, S.; Gillen, J. G.; Murray, C. B.; Kagan, C. R. Bandlike Transport in Strongly Coupled and Doped Quantum Dot Solids: A Route to High-Performance Thin-Film Electronics. Nano Lett. 2012, 12, 2631-2638.

(40) Balazs, D. M.; Matysiak, B. M.; Momand, J.; Shulga, A. G.; Ibáñez, M.; Kovalenko, M. V.; Kooi, B. J.; Loi, M. A. Electron Mobility of $24 \mathrm{~cm}^{2} \mathrm{~V}^{-1} \mathrm{~S}^{-1}$ in PbSe Colloidal-Quantum-Dot Superlattices. Adv. Mater. 201830, 1802265,.

(41) Gilmore, R. H.; Winslow, S. W.; Lee, E. M. Y.; Ashner, M. N.; Yager, K. G.; Willard, A. P.; Tisdale, W. A. Inverse Temperature Dependence of Charge Carrier Hopping in Quantum Dot Solids. ACS Nano 2018, 12, 7741-7749.

(42) Gao, J.; Zhang, J.; van de Lagemaat, J.; Johnson, J. C.; Beard, M. C. Charge Generation in PbS Quantum Dot Solar Cells Characterized by Temperature-Dependent Steady-State Photoluminescence. ACS Nano 2014, 8, 12814-12825.

(43) Zhang, J.; Tolentino, J.; Smith, E. R.; Zhang, J.; Beard, M. C.; Nozik, A. J.; Law, M.; Johnson, J. C. Carrier Transport in PbS and PbSe QD Films Measured by Photoluminescence Quenching. J. Phys. Chem. C 2014, 118, 16228-16235.

(44) Szendrei, K.; Speirs, M.; Gomulya, W.; Jarzab, D.; Manca, M.; Mikhnenko, O. V.; Yarema, M.; Kooi, B. J.; Heiss, W.; Loi, M. A. Exploring the Origin of the Temperature-Dependent Behavior of $\mathrm{PbS}$ Nanocrystal Thin Films and Solar Cells. Adv. Funct. Mater. 2012, 22, $1598-1605$

(45) Chuang, C.-H. M.; Maurano, A.; Brandt, R. E.; Hwang, G. W.; Jean, J.; Buonassisi, T.; Bulović, V.; Bawendi, M. G. Open-Circuit Voltage Deficit, Radiative Sub-Bandgap States, and Prospects in Quantum Dot Solar Cells. Nano Lett. 2015, 15, 3286-3294.

(46) Stavrinadis, A.; Pradhan, S.; Papagiorgis, P.; Itskos, G.; Konstantatos, G. Suppressing Deep Traps in PbS Colloidal Quantum Dots via Facile Iodide Substitutional Doping for Solar Cells with Efficiency > 10\%. ACS Energy Lett. 2017, 2, 739-744.

(47) Moreels, I.; Lambert, K.; Smeets, D.; De Muynck, D.; Nollet, T.; Martins, J. C.; Vanhaecke, F.; Vantomme, A.; Delerue, C.; Allan, G.; Hens, Z. Size-Dependent Optical Properties of Colloidal PbS Quantum Dots. ACS Nano 2009, 3, 3023-3030.

(48) Voznyy, O.; Levina, L.; Fan, F.; Walters, G.; Fan, J. Z.; Kiani, A.; Ip, A. H.; Thon, S. M.; Proppe, A. H.; Liu, M.; Sargent, E. H. Origins of Stokes Shift in PbS Nanocrystals. Nano Lett. 2017, 17, 7191-7195.

(49) Kahmann, S.; Mura, A.; Protesescu, L.; Kovalenko, M. V.; Brabec, C. J.; Loi, M. A. Opto-Electronics of PbS Quantum Dot and Narrow Bandgap Polymer Blends. J. Mater. Chem. C 2015, 3, 54995505.

(50) Zaumseil, J.; Donley, C. L.; Kim, J.-S.; Friend, R. H.; Sirringhaus, H. Efficient Top-Gate, Ambipolar, Light-Emitting Field-
Effect Transistors Based on a Green-Light-Emitting Polyfluorene. Adv. Mater. 2006, 18, 2708-2712.

(51) Loi, M. A.; Rost-Bietsch, C.; Murgia, M.; Karg, S.; Riess, W.; Muccini, M. Tuning Optoelectronic Properties of Ambipolar Organic Light- Emitting Transistors Using a Bulk-Heterojunction Approach. Adv. Funct. Mater. 2006, 16, 41-47.

(52) Kim, D. K.; Lai, Y.; Vemulkar, T. R.; Kagan, C. R. Flexible, Low-Voltage, and Low-Hysteresis PbSe Nanowire Field-Effect Transistors. ACS Nano 2011, 5, 10074-10083.

(53) Chung, D. S.; Lee, J.-S.; Huang, J.; Nag, A.; Ithurria, S.; Talapin, D. V. Low Voltage, Hysteresis Free, and High Mobility Transistors from All-Inorganic Colloidal Nanocrystals. Nano Lett. 2012, 12, $1813-1820$.

(54) Osedach, T. P.; Zhao, N.; Andrew, T. L.; Brown, P. R.; Wanger, D. D.; Strasfeld, D. B.; Chang, L.-Y.; Bawendi, M. G.; Bulović, V. BiasStress Effect in 1,2-Ethanedithiol-Treated PbS Quantum Dot FieldEffect Transistors. ACS Nano 2012, 6, 3121-3127.

(55) Nugraha, M. I.; Häusermann, R.; Bisri, S. Z.; Matsui, H.; Sytnyk, M.; Heiss, W.; Takeya, J.; Loi, M. A. High Mobility and Low Density of Trap States in Dual-Solid-Gated PbS Nanocrystal FieldEffect Transistors. Adv. Mater. 2015, 27, 2107-2112.

(56) Zhang, Y.; Zherebetskyy, D.; Bronstein, N. D.; Barja, S.; Lichtenstein, L.; Alivisatos, A. P.; Wang, L.-W.; Salmeron, M. Molecular Oxygen Induced In-Gap States in $\mathrm{PbS}$ Quantum Dots. ACS Nano 2015, 9, 10445-10452.

(57) Boehme, S. C.; Azpiroz, J. M.; Aulin, Y. V.; Grozema, F. C.; Vanmaekelbergh, D.; Siebbeles, L. D. A.; Infante, I.; Houtepen, A. J. Density of Trap States and Auger-Mediated Electron Trapping in CdTe Quantum-Dot Solids. Nano Lett. 2015, 15, 3056-3066.

(58) Mott, N. F. Conduction in Glasses Containing Transition Metal Ions. J. Non-Cryst. Solids 1968, 1, 1-17.

(59) Rubinger, R. M.; Ribeiro, G. M.; Oliveira, A. G. de; Albuquerque, H. A.; Silva, R. L. da; Rubinger, C. P. L.; Rodrigues, W. N.; Moreira, M. V. B. Temperature-Dependent Activation Energy and Variable Range Hopping in Semi-Insulating GaAs. Semicond. Sci. Technol. 2006, 21, 1681.

(60) Muccini, M.; Toffanin, S. Photonic Properties of OLETs. In Organic Light-Emitting Transistors; Wiley-Blackwell, 2016; pp 201241.

(61) Gao, J.; Johnson, J. C. Charge Trapping in Bright and Dark States of Coupled PbS Quantum Dot Films. ACS Nano 2012, 6, 3292-3303. 